\title{
The Preparation of Ultrathin Frozen Sections for Immunocytochemistry at the Electron Microscope Level
}

\author{
A. KENT CHRISTENSEN AND THOMAS E. KOMOROWSKI \\ Department of Anatomy and Cell Biology, The University of Michigan \\ Medical School, Ann Arbor, Michigan 48109
}

\begin{abstract}
KEY WORDS Ultrathin frozen sections, Electron microscopy, Immunocytochemistry, Cryobiology
\end{abstract}

\begin{abstract}
A description of ultrathin frozen sectioning is presented. Small pieces of lightly-fixed but unembedded tissue are cryoprotected in $80 \%$ sucrose, mounted on chucks and frozen in liquid nitrogen. Frozen sections approximately $100 \mathrm{~nm}$ thick for electron microscopy are cut at about $-90^{\circ} \mathrm{C}$, using a cryosectioning unit mounted on a conventional ultramicrotome. Sections are picked up from the surface of the knife with a droplet of $80 \%$ sucrose, and are applied to membrane-coated EM grids at room temperature. The mounted sections are then used for EM immunocytochemistry. Essentially the same sectioning procedure can provide frozen sections $1 \mu \mathrm{m}$ thick for light microscope immunocytochemistry.
\end{abstract}

Ultrathin frozen sections provide a favorable basis for immunocytochemistry at the electron microscope (EM) level, and this approach avoids many of the problems inherent in current preembedding and postembedding procedures for EM immunocytochemistry.

The method described here for cutting ultrathin frozen sections was developed by Christensen (1971). The quality and consistency of the morphology obtained was greatly improved by the subsequent development (Tokuyasu, 1973) of sucrose cryoprotection and of a very effective means of picking up sections, spreading them and applying them to grids. Still further improvements came from methods of staining, postembedding, and adaptations for immunocytochemistry (Tokuyasu, 1978, 1980; Tokuyasu and Singer, 1976; Geuze et al., 1981; and others). The preparation of ultrathin frozen sections and their use in immunocytochemistry has been reviewed by Griffiths et al. (1983), Tokuyasu (1984), and Ivanov et al. (1984).

The equipment for ultrathin frozen sectioning utilized here is the Dupont/Sorvall FTS System, which was based on a unit (Fig. 1) developed by Christensen (1971) for use on the Sorvall MT-2B ultramicrotome. Three other more recent systems are also available: (1) the Dupont/Sorvall FS1000 Cryo Sectioning System (for the MT6000, MT5000, and MT-2B Ultramicrotomes), (2) the ReichertJung FC4 Low-temperature Sectioning System (for the Ultracut E Ultramicrotome), and
(3) the LKB CryoNova (for the Ultratome Nova). The method for cutting ultrathin frozen sections on these other instruments is similar to the one described here.

\section{Tissue preparation}

1. If possible, organs should be fixed by vascular perfusion. Perfuse-fix for about 0.5 1 hour, then cut the tissue into slices $1-2 \mathrm{~mm}$ thick with a razor blade. Perfusion or immersion fixation may be followed by a wash in phosphate buffered saline (PBS) overnight in the refrigerator, or by further fixation overnight (see below).

2 . You may need to try several fixatives to determine which is best for the antigen you are trying to localize, since antigens differ greatly in their stability under fixation. Paraformaldehyde is a relatively gentle fixative but doesn't preserve well for electron microscopy, and the fixation is reversible and therefore doesn't last. Glutaraldehyde yields good ultrastructural preservation, but commonly

Received January 17, 1985; accepted February 2, 1985.

Address reprint requests to Dr. A. Kent Christensen, Department of Anatomy and Cell Biology, Medical Science II Building, The University of Michigan Medical School, Ann Arbor, MI 48109.

This article was originally prepared as a handout for a workshop entitled Cryoultramicrotomy and Immunolabeling of U1trathin Sections, held in conjunction with the annual meetings of the American Society for Cell Biology, Kansas City, Missouri, on November 12, 1984. Dr. Christensen was chairman and moderator of the workshop and was one of nine speakers. The work shop was organized by the ASCB Education Committee. 


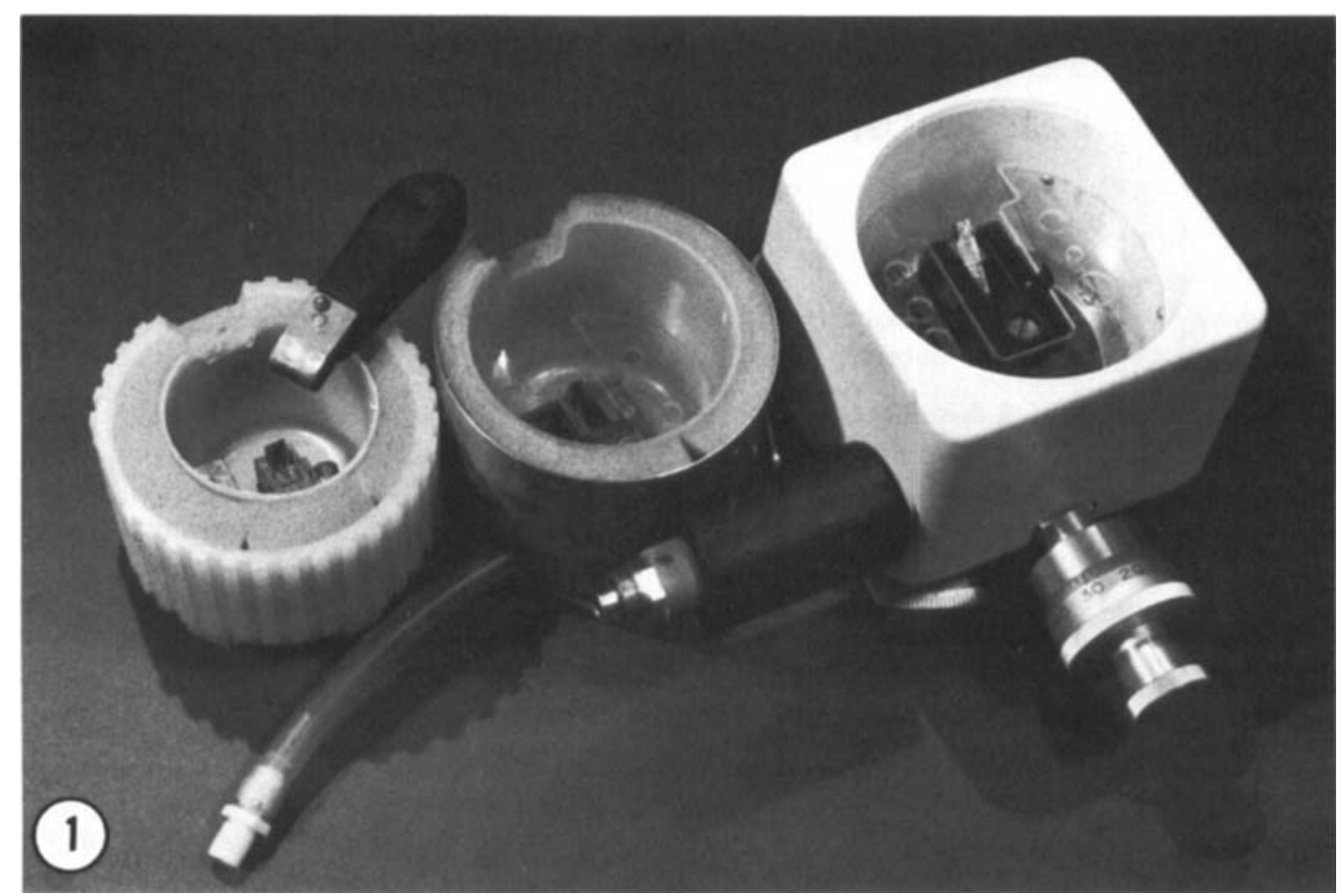

Fig. 1. Stages in the development of the cryosectioning bowl. The original prototype (left), dating from 1966, was made from the inverted cap of a 5-gal plastic jug, with foam rubber and a plastic beaker placed inside to form an insulated wall. An improvised knifeholder was mounted in the bottom of the bowl. Liquid nitrogen or freon 22 was poured into the bowl to a level below the knife edge, and the bowl thus acted as a dewar. The frozen tissue was mounted on a copper chuck at the lower end of an ebony bar (here shown balanced on the wall) that extended down into the bowl from the cutting arm of the ultramicrotome, bringing the tissue into proper position at the knife edge for cutting in the cold atmosphere. A later model (center) had a stainless steel beaker as the outer layer and had a more stable knifeholder. It was used in the same manner as the previous model until 1968, when a new method of cooling the bowl was adopted, using cold nitrogen gas that entered the bowl through a tube. About that time the Sorvall Company became interested in the device, and their production model (right), which was called the Frozen Thin Sectioner (FTS), became available in 1970. reduces antigenicity and produces cross-linking in the tissue that may impede access of antibodies to the antigens. As a consequence, glutaraldehyde must usually be used in low concentration, if at all. A good fixative to begin with is $4 \%$ formaldehyde and $0.2 \%$ glutaraldehyde in $0.1 \mathrm{M}$ phosphate or cacodylate buffer $(\mathrm{pH} \mathrm{7.4)}$ at room temperature. We use purified formaldehyde supplied in sealed vials (20\%, Ladd), but it can also be prepared from paraformaldehyde.

For problem antigens you may want to try other fixatives: (1) $8 \%$ formaldehyde (Slot and Geuze, 1982), (2) picric-acid formaldehyde (Stefanini et al., 1967), (3) periodate-lysineparaformaldehyde (McLean and Nakane, 1974), (4) acrolein (King et al., 1983), (5) ethyl acetimidate followed by glutaraldehyde (Tokuyasu and Singer, 1976; Tokuyasu, 1980).
3. If the initial fixative contained formaldehyde, it may be advantageous to leave the tissue slices overnight in $4 \%$ formaldehyde in $0.1 \mathrm{M}$ phosphate buffer in the refrigerator. This postfixation is said (Eldred et al., 1983) to be more effective if the $\mathrm{pH}$ of the fixative is basic, for example, $\mathrm{pH} 10.4$ buffered with $0.1 \mathrm{M}$ sodium bicarbonate (some antigens may not tolerate this $\mathrm{pH}$ ). If the initial fixative included glutaraldehyde, you may also want to treat the tissues the next morning with borohydride (Eldred et al., 1983), which reduces residual aldehydes and may also partially restore antigenicity by reducing Schiff bases (carbon-nitrogen double bonds) that can be formed when glutaraldehyde reacts with free amino groups on proteins. Borohydride treatment also reduces tissue autofluorescence in light microscope fluorescence im- 
munocytochemistry. Use $1 \%$ sodium borohydride in PBS for $30 \mathrm{~min}$ (room temperature) followed by a wash in two changes of PBS over 1 hour.

4. After PBS washes, the tissue can be taken in graded steps into $2.3 \mathrm{M}(80 \%)$ sucrose in PBS (room temperature) as a cryoprotectant (Tokuyasu, 1973). We use a sequence of PBS to 2.3 M sucrose ratios (30 min each) of $2: 1,1: 1,1: 2$, then straight $2.3 \mathrm{M}$.

5 . After either step 3 or step 4 above, the tissue may be stored in PBS or 2.3 M sucrose in the refrigerator for a week or more with little loss of antigenicity (depending on the antigen). If desired, the solution can include $0.5 \%$ formaldehyde to sustain fixation during storage (probably unnecessary if the original fixative involved substantial glutaraldehyde).

Setting up the Sorvall FTS unit (Fig. 2)

1. Remove the conventional knife stage and the specimen holder mount from the Sorvall MT-2B ultramicrotome (if they are on). Replace the stage with the FTS stage, and clamp the lever (on left side of microtome base) down tightly. Make sure that both spring-loaded levers (at left and front on the base of the bowl) and the coarse advance lock (knob at left side) are loose.

2. Replace the specimen holder mount with the rear portion of the FTS specimen holder assembly. Then insert the front portion, extending down into the FTS bowl, and clamp the lever tightly. Connect the temperature cable from specimen holder to the Sorvall LTC-2 Low Temperature Controller ("sensor" socket). Set the LTC-2 (Fig. 3) to the desired temperature (red needle), usually about $-90^{\circ} \mathrm{C}$ for $\mathrm{EM}$ sections. In order to know the actual environmental temperature in which the cutting is taking place, we install a copper-constantan thermocouple alongside the knife edge and run the connecting wire to a digital thermometer (Fig. 3) (Digicator model 400B, from Omega Engineering, 1 Omega Drive, Box 4047, Stamford, CT 06907; thermocouple from same source). The wire from the thermocouple (Fig. 4) needs to run horizontally for $2-3$ inches before rising out of the bowl, to avoid the influence of room temperature.

3. Put the heater rod down into a Dewar of liquid nitrogen (Fig. 2) and press the stopper to obtain a good seal. Be sure the tube is connected properly with the FTS bowl and that the cable is plugged into the LTC-2 "load" socket (Fig. 3). About 15-20 minutes before you intend to begin cutting sections, turn on the LTC-2 by pressing the "power" button (red light will come on).

4. Prepare glass knives on an LKB Knifemaker. Use $45^{\circ}$, and set the Knifemaker so the break occurs parallel with and very close to the corners of the 1-in glass square. It has been reported (Roberts, 1975) that a coating of tungsten improves the cutting characteristics of glass knives. Diamond knives can be used, but glass is adequate (and mistakes are less expensive).

5. Loosen the knifeholder screw (Fig. 4) with a screwdriver and insert a glass knife. Push the knife forward to the proper position and then tighten the holder screw. The cutting angle should be set at about $6^{\circ}$ (each unit is $2^{\circ}$ ). (The knife receptacle on the FTS knifeholder needs some adjustment to maintain a standard 1-in LKB knife at the proper height for cutting. We routinely place a small plate of metal or plastic about $3.5 \mathrm{~mm}$ thick in the bottom of the receptacle, to hold the knife up in proper position; the two small screws that are provided for that purpose should be removed]. Sections will be cut with a dry knife (no flotation medium or "boat" is used).

6. Cover the FTS bowl with a piece of flat plastic while it is coming to the desired temperature and at other times when you are not actually cutting sections. During sectioning you can either remove the cover or slide it back to expose part of the opening of the bowl.

\section{Ultrathin frozen sections (UFS) for electron microscopy}

1. For sectioning, prepare tissue blocks (about $0.5 \mathrm{~mm}$ in smallest diameter) of sucrose-treated tissue, using a sharp razor blade while observing under a dissecting microscope (adjust substage mirror for optimal viewing). Place a tissue block on a copper chuck and orient it properly so the cutting face will be about $0.5 \mathrm{~mm}$ in diameter. Gently blot off excess fluid with filter paper or Kimwipe. We routinely place a little mounting medium (Tissue-Tek O.C.T. Compound, LabTek Products, Naperville, IL) on the chuck before the tissue block is mounted; this acts as a base to support the tissue and attach it firmly to the chuck. For freezing, grasp the chuck with forceps and immerse it in liquid nitrogen (in a small Styrofoam receptacle) until rapid bubbling ceases. Frozen tissue blocks on chucks can be stored in small cans 


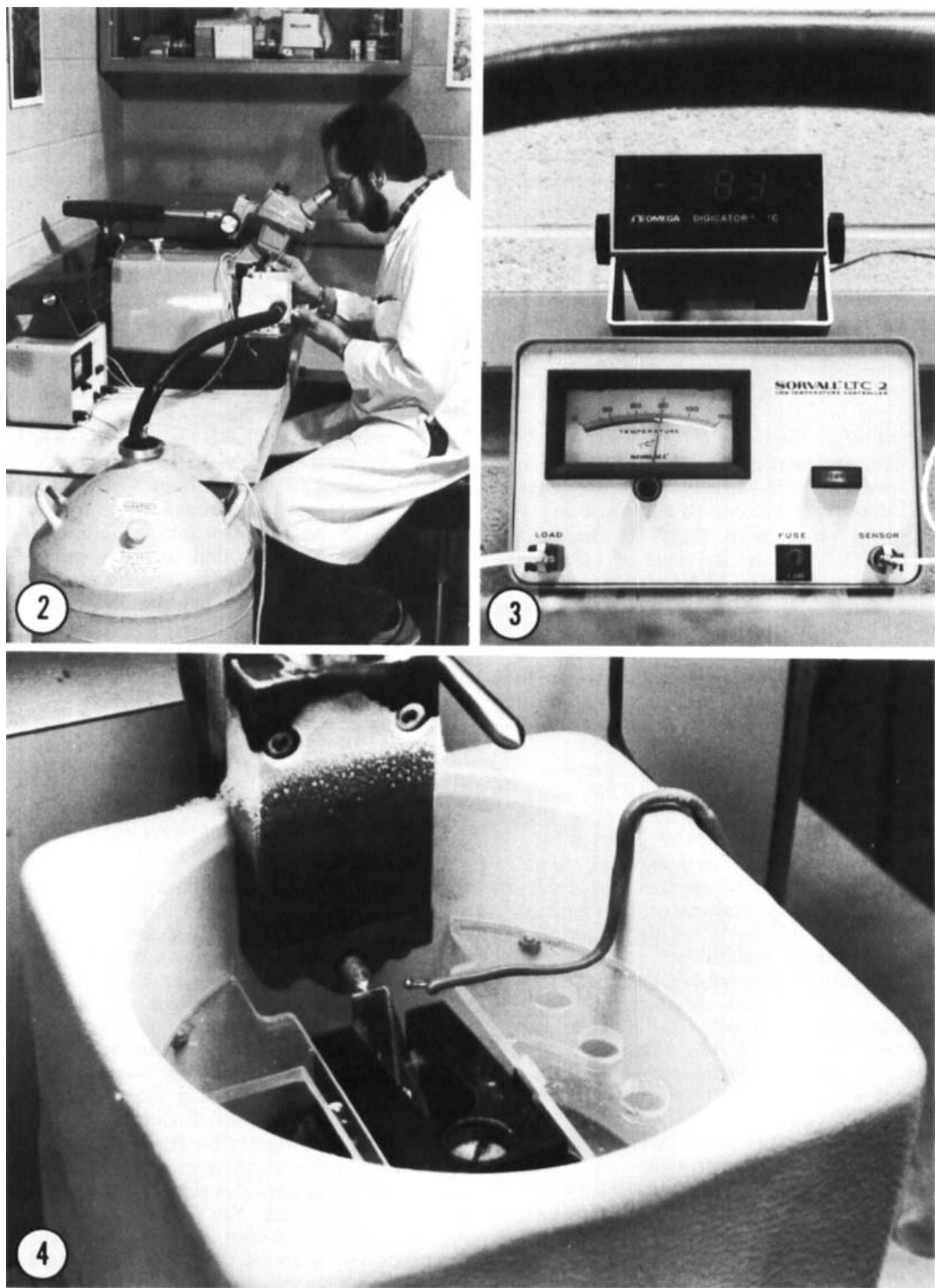


in a liquid nitrogen refrigerator indefinitely without antigen deterioration; chucks must be supported in the cans to keep them from rolling around, which will often break the tissue blocks off the chucks.

2. Grasp a chuck with forceps and transfer it quickly from liquid nitrogen to the FTS bowl (Fig. 4), where it is inserted into the hole in the specimen holder. You will have to determine empirically how far out it should extend to allow proper cutting: if the chuck extends too far out, then the specimen holder may scrape on the inside back surface of the cutting bowl; if too far in, then the knife advance may not be sufficient to reach it. Orient the chuck as desired and then clamp tightly (knob on top of specimen holder assembly).

3. If desired, the block can be "faced," using the right- or left-hand third of the knife edge, by cutting several thick sections (about $0.5 \mu \mathrm{m}$ ). You can then back off slightly, move to the optimal region of the knife edge (mid-

Fig. 2. Overall view of equipment for cutting ultrathin frozen sections. The Dupont/Sorvall FTS bowl is mounted on the stage of an MT-2B Ultramicrotome. Cold nitrogen gas, generated in a dewar (foreground), passes through an insulated tube to enter the bowl. Within the dewar, the production of cold gas is regulated by current through a heater suspended in liquid nitrogen. The current, in turn, is controlled by the Low Temperature Con troller (LTC-2), on the table just to the left of the microtome. The temperature within the bowl is deter mined by the rate of gas flow from the dewar. Since the gas is warmed to some extent during its passage through the insulated tube, a faster rate of flow means less time in the tube and therefore a colder temperature in the bowl.

Fig. 3. The Dupont/Sorvall Low Temperature Controller (LTC-2) used to set and maintain temperatures in the bowl. The wire at left (marked "load") provides current to the heater in the dewar. The wire at right (marked "sensor") brings in temperature readings from a sensor located in the copper chuckholder within the bowl. Any desired temperature can be set with a red needle on the dial (here set at $-90^{\circ} \mathrm{C}$ ), and the actual temperature, indicated by a black needle, will then come to that level A digital thermometer has been placed on top of the LTC-2 unit and reads a thermocouple situated near the knife edge in the bowl (see next figure).

Fig. 4. Interior of the FTS bowl set for cutting at $-90^{\circ} \mathrm{C}$. A glass knife is in place, and a tissue specimen, mounted at the tip of a copper chuck, is near the knife edge in position for cutting. A thermocouple, at the end of a wire entering the bowl from the right, is suspended near the knife edge, measuring environmental temperature where cutting actually occurs. The cold nitrogen gas that fills the bowl is heavier than air and therefore excludes it from the bowl (note that the frostline does not extend appreciably below the rim). dle third), and then advance carefully for ultrathin sections. We generally do not "face" the block, but rather begin directly with ultrathin sections on the middle third of the knife edge.

4. Cut ultrathin frozen sections (UFS) (Fig. 5). Set a cutting thickness of about 50-100 nm (8-9 on "thickness" dial; 10 on top knob of MT-2B). Use a slow cutting speed, either in manual mode (with a slow pass and a rapid return) or with the instrument set for automatic cutting (about 0.63 on "speed" dial). Bring knife somewhat close to tissue with coarse advance knob (lower front of bowl), then tighten coarse advance lock (knob at left of bowl). Advance still closer with fine advance knob $(0.5-\mu \mathrm{m}$ increment per pass) until you either see a hint of sectioning or are confident that sectioning is imminent, then allow normal passes until ultrathin sectioning begins. Sections accumulate on the surface of the knife (Fig. 5). Use an eyelash probe (Fig. 6), mounted at the end of a wooden stick or pasteur pipette, to prevent sections from swinging back over the knife edge, and also, if possible, to move the sections to favorable positions on the knife surface for pickup. If conditions are such that sections move erratically because of static charge, a conventional antistatic device (ionization source) may prove helpful.

5. Cut as many sections as possible without stopping. After you stop cutting for a few moments, it is necessary to be cautious when you begin sectioning again, since minor fluctuations of temperature at different levels in the bowl can cause the chuck and/or specimen holder to expand or contract slightly, yielding a thick section or no section when you start up again. This effect is minimized if you always stop with the chuck at the level of the knife edge, in the back phase of the cutting cycle. Otherwise, back off $0.5-1 \mu \mathrm{m}$ (with fine advance knob) and approach again. Variations in temperature or in tissue consistency can also give rise to "skipping." When sections become unacceptable at a given place on the knife edge, you can move the knife over slightly to a new area (using knob at lower right on bowl). Knives can be changed during a cutting session without warming the bowl; merely loosen the large screw, lift out the old knife, insert the new knife, tighten the screw, and wait a few minutes for thermal equilibration.

6 . The cutting temperature has an effect on section quality. If the temperature is too cold, then sections tend to be brittle and frag- 

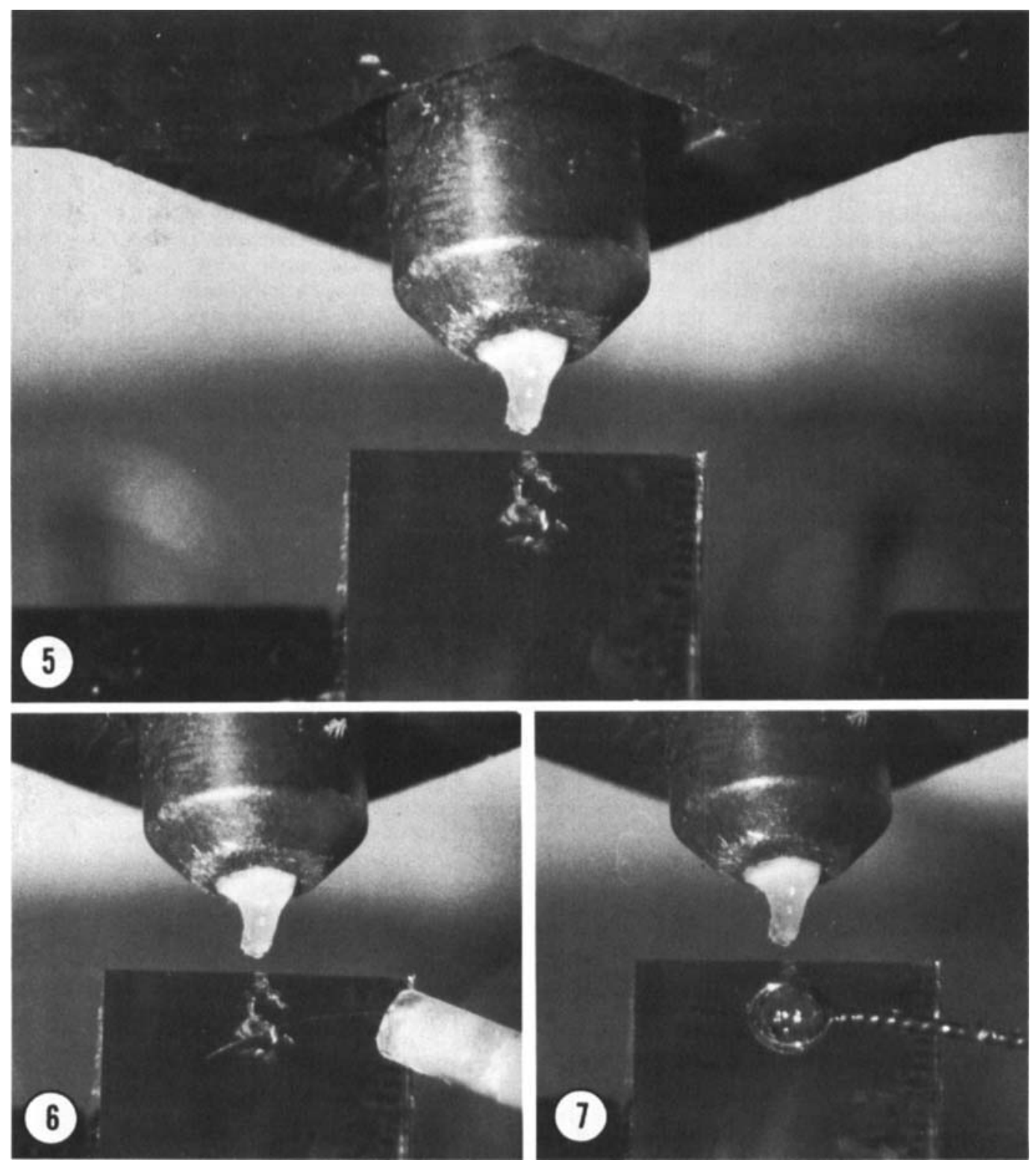

Fig. 5. Specimen and knife seen through the Bausch \& Lomb viewing microscope of the ultramicrotome during a cutting session. The temperature is set at $-90^{\circ} \mathrm{C}$. Note ultrathin frozen sections lying dry on the surface of the knife, awaiting pickup.
Fig. 6. An eyelash probe can be used to move or otherwise manipulate the sections on the surface of the knife.

Fig. 7. Sections are picked up on the underside of a droplet of $2.3 \mathrm{M}$ sucrose, suspended in a wire loop. 
mented, while sections may appear "mushy" during cutting if the temperature is too warm. Slight changes of temperature $\left(2-3^{\circ}\right.$ at a time, with the red needle on the LTC-2) may correct these problems. In general, the thinner the sections you are trying to cut, the colder the temperature will need to be. If your research requires especially thin ultrathin frozen sections, for example, $50 \mathrm{~nm}$, then you may need to cut with the LTC-2 set at about $-110^{\circ} \mathrm{C}$. Packing some additional insulation (not too much) around the insulated tube connecting the dewar to the bowl may reduce the velocity of nitrogen gas necessary to maintain these colder temperatures.

7. Although frost is generally negligible within the bowl, very fine frost can form on the knife edge and may make cutting less effective. It can be removed by running the wedge-shaped end of an orangewood or balsa stick gently along the knife edge (this can even be done from time to time between strokes during cutting). Try to keep room humidity down, if possible, and avoid breathing directly into the bowl. A small plastic plate can be suspended from the viewing $\mathrm{mi}$ croscope to protect the bowl from the operator's breath.

8. Pick up the sections (Tokuyasu, 1973) with a droplet of $2.3 \mathrm{M}$ sucrose (in PBS) held in a small wire loop (Fig. 7) about $1.25 \mathrm{~mm}$ inside diameter, mounted at the end of a wooden stick. Make this loop with fine nichrome or platinum wire, using the end of a Pasteur pipette as a template. Bring the loop (with droplet) into the bowl, and before the droplet freezes touch it to a group of sections but not the knife edge, if you can avoid it. The sections will adhere to the bottom of the droplet, which soon freezes. Retract the droplet from the bowl. With a little practice, sections can be picked up between sectioning passes without stopping the microtome.

9. Nickel or copper grids should previously have been coated with Formvar membranes. Stabilize the membranes with a carbon coat, which must then be made hydrophilic by ionization under a glow discharge ring within a vacuum evaporator (about 30 seconds). We use 200-mesh grids but others recommend 100 mesh hexagonal grids.

10. When you remove the frozen droplet (bearing the sections) from the FTS bowl, let the droplet melt, and after allowing a few seconds for the sections to spread, touch the underside of the droplet to the center of a Formvar-coated grid (Fig. 8). The sections will attach firmly to the membrane. Since the concentrated sucrose dries very slowly, you can accumulate several grids with droplets on them (Fig. 8) before carrying them on to further steps. Do not allow the sections to dry out at any time during this or subsequent procedures.

11. Float the grids face down on $2 \%$ gelatin in PBS (room temperature) for at least 5 minutes to wash out the sucrose. If necessary, grids can be stored on the $2 \%$ gelatin for several hours in the refrigerator. Finally, the grids are carried through the series of steps for whichever EM immunocytochemistry procedure has been selected.

\section{Frozen sections for light microscopy}

1. The preparation of frozen sections for light microscope (LM) immunocytochemistry is essentially the same as the procedure for ultrathin frozen sections, described above. In the following description, some of the differences will be pointed out.

2 . We generally utilize the same perfusionfixed tissue for both LM and EM immunocytochemistry. Preparation of the tissue blocks, mounting on chucks, freezing, transfer to the FTS bowl, and setup of the FTS unit for cutting are the same, except that the tissue blocks can be larger (up to 1-2 mm in diameter), the cutting temperature set on the LTC2 is warmer (about $-50^{\circ} \mathrm{C}$ ), and section thickness is usually $0.5-1 \mu \mathrm{m}$ (although it can be up to $2-3 \mu \mathrm{m}$; these thicker sections require a warmer cutting temperature, about $-35^{\circ} \mathrm{C}$ ). If you are planning to cut both EM and LM frozen sections from the same tissue block, it is best to cut the ultrathin sections first, then allow the bowl to warm (covered with a flat plastic plate) to the proper temperature for the LM sections. The reason for this sequence is that recrystallization (substantial growth of the ice crystals within the frozen tissue) is accelerated at warmer temperatures, even with sucrose cryoprotection. Tissue blocks will become white, rather than the usual translucent gray, as serious recrystallization occurs.

3. The block should be "faced" by cutting sections on the right- or left-hand third of the knife edge (using the fine advance knob at lower middle of bowl) until an acceptable face is formed. Then shift to the middle third of the knife edge for sectioning.

4. Cut frozen sections. It is probably easiest to use the fine advance knob to set the thickness for each section. Each small division on 


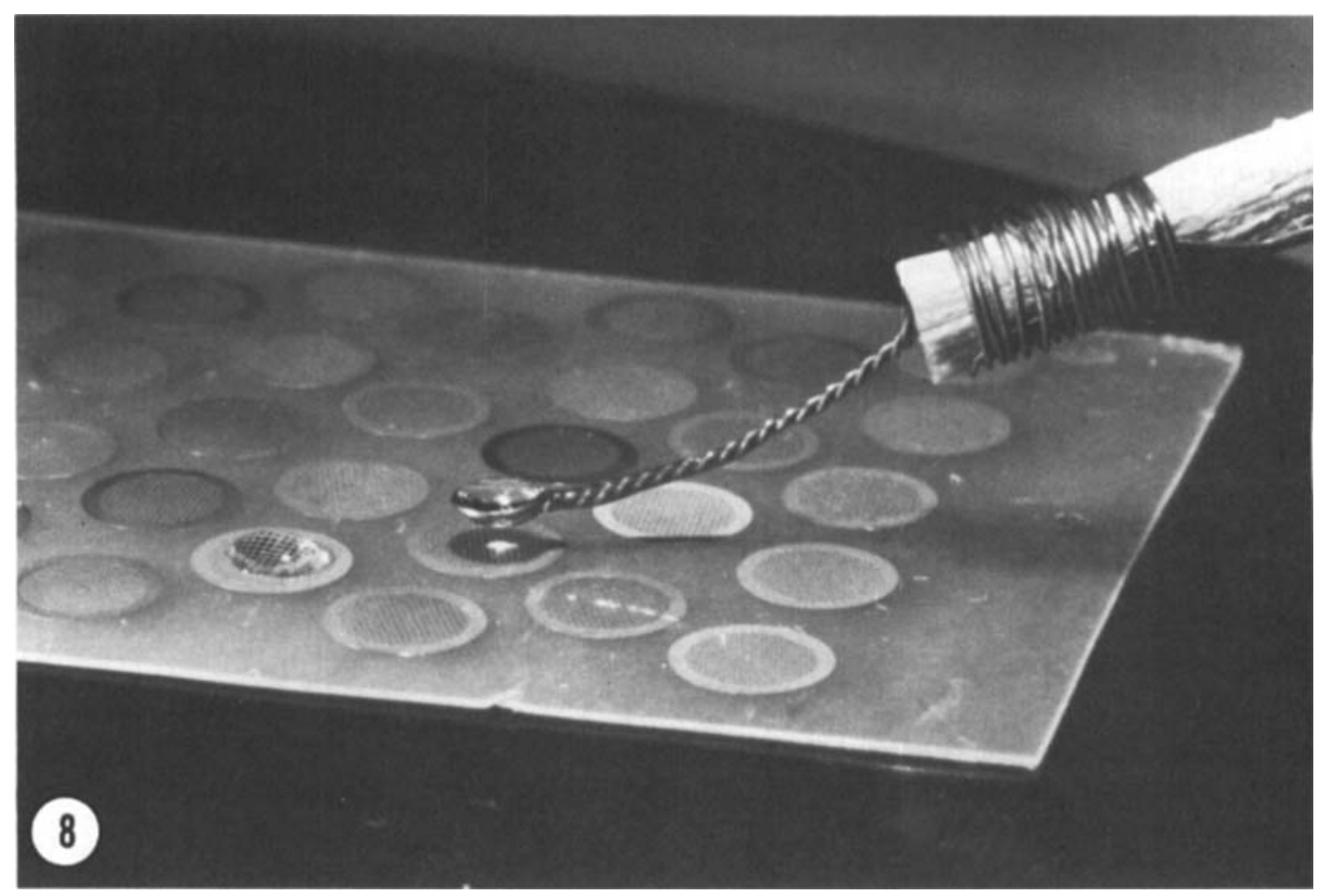

Fig. 8. After being removed from the bowl, the droplet (bearing the sections) is touched to the middle of an EM grid, and the sections attach firmly to the grid membrane. Another grid, to the left, has already received a droplet.

this knob represents $1 \mu \mathrm{m}$. We routinely use $1-\mu \mathrm{m}$ sections for immunofluorescence.

5. Pretreat clean $1 \times 3$-in glass slides or coverslips (on which you intend to mount the sections) with $0.01 \%$ poly-L-lysine (Sigma, St. Louis, MO) in distilled water for 1 hour in a coplin or columbia jar and then air dry. This coating helps bind the sections to the glass (Mazia et al., 1975).

6. Pick up sections or ribbons off the glass knife with a droplet of $2.3 \mathrm{M}$ sucrose suspended in a loop $2-3 \mathrm{~mm}$ in inner diameter. Before trying to pick up sections, it is necessary to leave the droplet in the cold atmosphere of the bowl until small white frozen areas appear on its surface. Then gently touch the droplet to the sections. If the touch is too early, then part of the droplet may be left on the knife; if too late, then the sections may not adhere.

7. Remove the droplet (bearing the sections) from the bowl. A few seconds after it melts, touch the droplet to a polylysine-treated slide or coverslip, and the sections will bind to the surface. Before touching the droplet to the slide, it may be advantageous to observe the droplet under a dissecting microscope, to ascertain the position of the sections on the undersurface of the droplet, so the droplet can be oriented properly as it is touched to the slide. More than one droplet with sections can be placed on the same slide, in case you want to run a simultaneous control along with the experimental. A circle can be drawn around clusters of sections with a diamond pencil, to mark their position and to form a boundary for subsequent droplets of immunocytochemical reagents.

8. Sections can then be carried through the various steps for immunocytochemistry at the light microscope level.

\section{EXAMPLES}

Examples of ultrathin frozen sections of rat testis, prepared in the manner described in this paper are shown in Figures 9 and 10. 


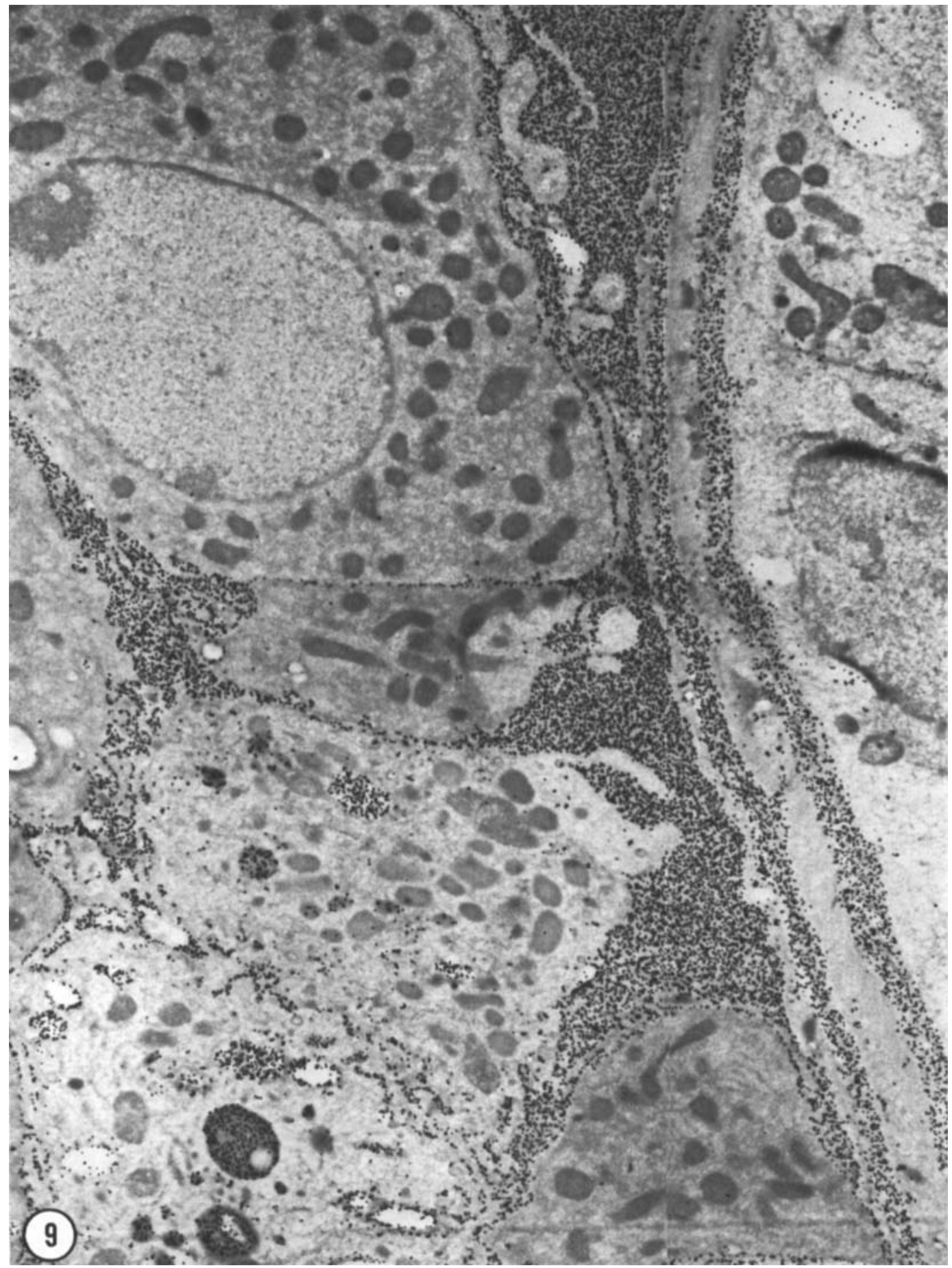

Fig. 9. Albumin activity, marked here by accumulations of gold particles (black dots), fills spaces between the seminiferous tubule at right, and the Leydig cell at upper left and macrophage at lower left. Magnification, $\times 7,900$. 


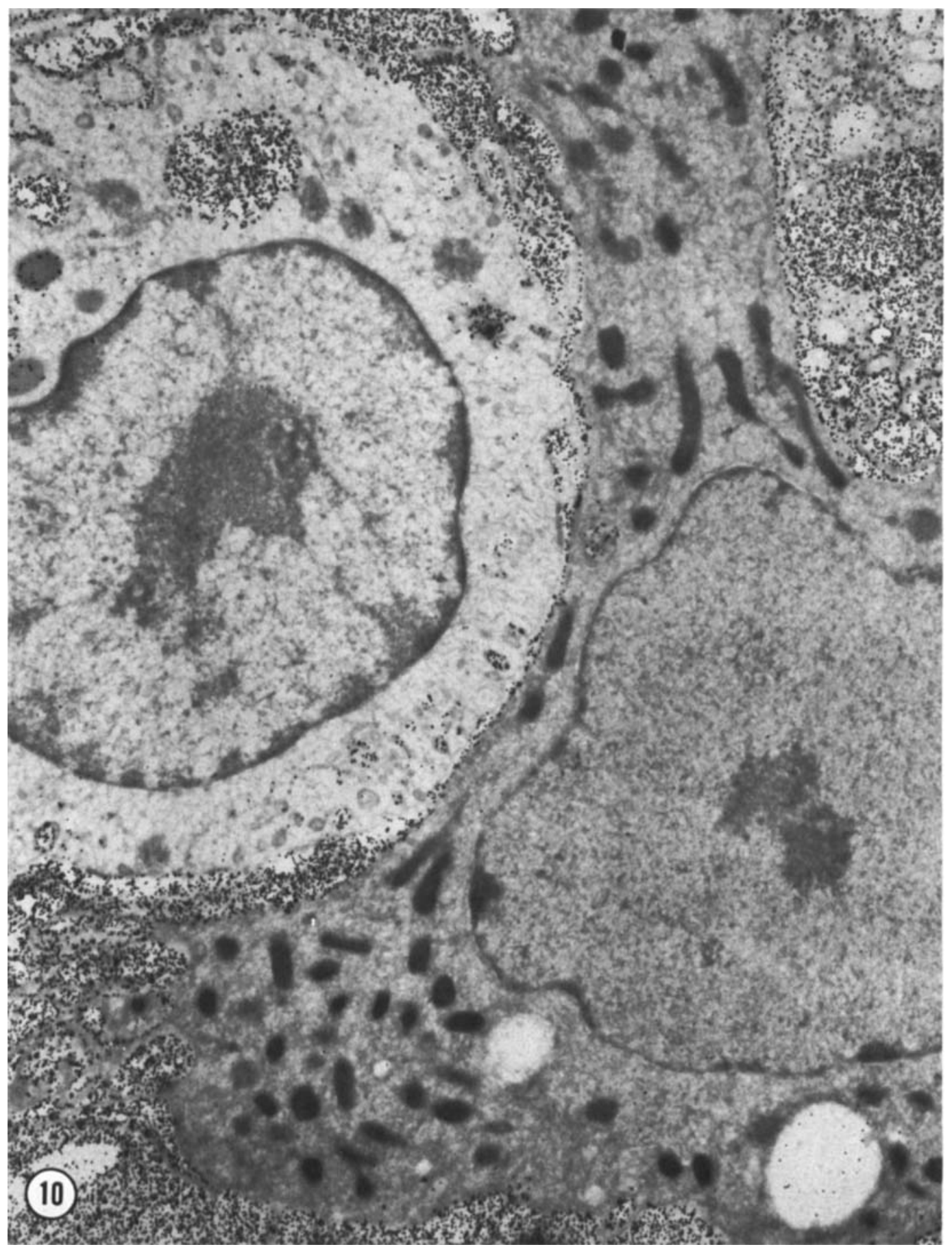

Fig. 10. Albumin activity is abundant in the interstitial space surrounding the macrophage at left and the Leydig cell at right. The macrophage shows evidence of endocytic uptake of albumin. Magnification, $\times 10,400$. 
The sections have been treated for gold particle immunocytochemistry, following procedures reviewed by Griffiths et al. (1983), Tokuyasu (1984), and Ivanov et al. (1984). Fixation was carried out by perfusion with $4 \%$ formaldehyde and $0.2 \%$ glutaraldehyde (buffered with phosphate), followed by basic formaldehyde and borohydride, as described in the text. The antigen is rat serum albumin. It has been localized here with a rabbit antibody, that was subsequently treated with $20 \mathrm{~nm}$ gold particles bearing a coat of goat antirabbit IgG (Janssen). For further details, see Christensen et al. (1985).

\section{REFERENCES}

Christensen, A.K. (1971) Frozen thin sections of fresh tissue for electron microscopy, with a description of pancreas and liver. J. Cell Biol., 51:772-804.

Christensen, A.K., Komorowski, T.E., Wilson, B., Ma, S.F., and Stevens, R.W., III. (1985) The distribution of serum albumin in the rat testis, studied by electron microscope immunocytochemistry on ultrathin frozen sections. Endocrinology, 116:1983-1996.

Eldred, W.D., Zucker, C., Karten, H.J., and Yazulla, S. (1983) Comparison of fixation and penetration enhancement techniques for use in ultrastructural immunocytochemistry. J. Histochem. Cytochem., 31:285292.

Geuze, H.J., Slot, J.W., van der Ley, P.A., and Scheffer, R.C.T. (1981) Use of colloidal gold particles in doublelabeling immunoelectron microscopy of ultrathin frozen tissue sections. J. Cell. Biol., 89:653-665.

Griffiths, G., Simons, K., Warren, G., and Tokuyasu, K.T. (1983) Immunoelectron microscopy using thin, frozen sections: Application to studies of the intracellular transport of Semiliki Forest virus spike glycoprotein. Methods Enzymol., 96:466-485.
Ivanov, I.E., Plesken, H., Sabatini, D.D., and Rindler, M.J. (1984) Immunolabelling of frozen thin sections and its application to the study of the biogenesis of epithelial cell plasma membranes. In J.D. Wade and S.A. Lewis (eds.): Current Topics in Membranes and Transport, Vol. 20. Molecular Approaches to Epithelial Transport. New York: Academic Press, pp. 199-216.

King, J.C., Lechan, R.M., Kugel, G., and Anthony, E.L.P. (1983) Acrolein: A fixative for immunocytochemical localization of peptides in the central nervous system. J. Histochem. Cytochem., 31:62-68.

Mazia, D., Schatten, G., and Sale, W. (1975) Adhesion of cells to surfaces coated with polylysine: Applications to electron microscopy. J. Cell Biol., 66:198-200.

McLean, I.W., and Nakane, P.K. (1974) Periodate-lysineparaformaldehyde fixative: A new fixative for immunoelectron microscopy. J. Histochem. Cytochem., 22:1077-1083.

Roberts, I.M. (1975) Tungsten coating - a method of improving glass microtome knives for cutting ultrathin frozen sections. J. Microsc. 103:113-119.

Slot, J.W., and Geuze, H.J. (1982) Ultracryomicrotomy of polyacrylamide embedded tissue for immunoelectron microscopy. Biol. Cell, 44:325-328.

Stefanini, M., De Martino, C., and Zamboni, L, (1967) Fixation of ejaculated spermatozoa for electron microscopy. Nature, 216:173-174.

Tokuyasu, K.T. (1973) A technique for ultracryotomy of cell suspensions and tissues. J. Cell Biol., 57:551-565.

Tokuyasu, K.T. (1978) A study of positive staining of ultrathin frozen sections. J. Ultrastruct. Res., 63:287307.

Tokuyasu, K.T. (1980) Immunochemistry on ultrathin frozen sections. Histochem. J., 12:381-403.

Tokuyasu, K.T. (1984) Immuno-cryoultramicrotomy. In J.M. Polak and I.M. Varndell (eds.) Immunolabeling for Electron Microscopy. New York: Elsevier, pp. 7182.

Tokuyasu, K.T., and Singer, S.J. (1976) Improved procedures for immunoferritin labeling of ultrathin frozen sections. J. Cell Biol., 71:894-906. 